\title{
Monitoring dengue outbreaks during COVID-19 crisis in dengue endemic countries
}

\author{
Asaduzzaman Miah \\ Associate Professor, Dept. of Entomology \\ Patuakhali Science and Technology University, Bangladesh
}

10.3396/ijic.v16i2.011.20

Keywords: COVID-19, dengue fever, outbreak, co-infection, Bangladesh

The COVID-19 has been declared as a global pandemic by the World Health Organization (WHO). ${ }^{1}$ During the COVID-19 crisis, dengue cases have increased especially in dengue-endemic countries. Several dengue-endemic countries in South Asia like the Philippines, Malaysia, Singapore, Thailand, Indonesia, Myanmar, India, Bangladesh, Pakistan, and South America like Brazil, Paraguay, Colombia are currently in the risk of dengue outbreak as dengue virus infection is increasing at an alarming rate. ${ }^{2}$ Most of these countries have a history of repeatedly occurring outbreaks of dengue. Interestingly all these countries are currently also suffering seriously from COVID-19. We are fighting against COVID-19, the situation may be more difficult to manage if the dengue outbreak is further added. The combination of COVID-19 and dengue is assumed as dangerous for the health system in any country. Moreover, concurrent outbreaks of dengue and COVID-19 may happen in these countries as both corona and dengue viruses are co-existing. For instance, the Philippines, Malaysia and Singapore are suffering currently from concurrent outbreaks of dengue and COVID-19. ${ }^{3}$

\section{Corresponding Author}

Asaduzzaman Miah

Dept. of Entomology, Patuakhali Science and Technology University, Dumki, Patuakhali,8602, Bangladesh

E-mail:mamiah81@yahoo.com 
During the COVID-19 crisis, there is a possibility to have co-infection of corona and dengue virus in these dengue-endemic countries as both the viruses are co-existing. ${ }^{4}$ Indeed, the co-infection cases will be increased if dengue outbreak occurs. Co-infection of corona and dengue has been reported recently in different dengue-endemic countries like Singapore, ${ }^{5}$ Thailand, ${ }^{6}$ India ${ }^{7}$ and Bangladesh. ${ }^{8}$ As an extremely unique occurrence, Singapore at first confirmed two patients who were co-infected with both corona and dengue virus. The report said that the co-infected people died in Thailand, India and Bangladesh. In this context, it is speculated that co-infection will occur accordingly in the other dengue-endemic countries. Besides, co-infection poses a challenge for accurate diagnosis and treatment, particularly when symptoms such as fever and aches can be similar for several viral diseases like dengue and COVID-19. The initial symptoms of dengue and COVID-19 disease are seen to be similar; the distinction can be made as the disease progresses. Hence it is difficult to distinguish between dengue and COVID-19 as their clinical and laboratory features are quite identical. In this regard, a study has been conducted in Singapore where it was reported that COVID-19 patients can produce false-positive results for dengue in the rapid serological tests. ${ }^{5}$ The new symptom of COVID-19 was discovered in the study where the patient was suspected of suffering from dengue fever. The report warned that COVID-19 patients were wrongly considered as dengue which resulted in a delay in diagnosis of corona infection and further spread of the virus. Therefore, co-infection may pose new challenges in the different dengue-endemic countries especially for health professionals.

The countries are at risk of possible co-infection and concurrent outbreaks in where COVID-19 and dengue are co-existing, and the viruses are co-circulating. It appears that several patients have been co-infected with corona and dengue virus and concurrent outbreaks have also been started. Thus, co-infection and concurrent outbreak trigger alert, especially in the dengue-endemic zones where dengue outbreak occurred repeatedly. For facing the upcoming dengue outbreak, special measures should be taken in these countries and therefore, an effective mosquito control program is strongly recommended.

\section{References}

1. World Health Organization. Coronavirus disease 2019 (COVID-19) Situation Report - 69. 29 March 2020. https://wwwwhoint/emergencies/diseases/novelcoronavirus-2019/situation-reports/. Accessed 10 June 2020.

2. European Centre for Disease Prevention and Control. Dengue worldwide overview. https://wwwecdceuropaeu/en/denguemonthly. Accessed 9 June 2020.

3. Southeast Asia at risk of missing coronavirus cases amid dengue outbreak: experts. https://wwwscmpcom/week-asia/ health-environment/article/3074496/. Accessed 10 June 2020.

4. Wu D, Lu J, Liu Q, Ma X, He W. To alert coinfection of COVID-19 and dengue virus in developing countries in the dengue-endemic area. Infect Control Hosp Epidemiol 2020; 1. https://doi.org/10.1017/ice.2020.187

5. Yan G, Lee CK, Lam LTM, et al. Covert COVID-19 and false-positive dengue serology in Singapore. Lancet Infect Dis 2020; 20(5): 536. https://doi.org/10.1016/S14733099(20)30158-4

6. Joob B, Wiwanitkit V. COVID-19 can present with a rash and be mistaken for dengue. J Am Acad Dermatol 2020; 82(5): e177. https://doi.org/10.1016/j.jaad.2020.03.036

7. Ayub J. Stung by dengue, patient dies of coronavirus co-infection in Bhopal. The Times of India. https:// timesofindia.indiatimes.com/city/bhopal/stung-by-denguepatient-dies-of-coronavirus-co-infection-in-bhopal/ articleshow/75330826.cms. Accessed 10 June 2020.

8. Report SO. A double struggle: Home ministry PRO battling Covid-19, dengue. The Daily Star. https://www.thedailystar. net/coronavirus-deadly-new-threat/news/double-strugglehome-ministry-pro-battling-covid-19-dengue-1905013. Accessed 10 June 2020. 